\title{
Elastic Domains Regulate Growth and Organogenesis in the Plant Shoot Apical Meristem
}

Daniel Kierzkowski, ${ }^{{ }^{*}}{ }^{*}$ Naomi Nakayama, ${ }^{{ }_{*}}{ }^{*}$ Anne-Lise Routier-Kierzkowska, ${ }^{{ }^{1_{*}}}$ Alain Weber, ${ }^{1 *}$ Emmanuelle Bayer, ${ }^{2}$ Martine Schorderet, ${ }^{3}$ Didier Reinhardt, ${ }^{3}$ Cris Kuhlemeier, ${ }^{1}$ Richard S. Smith ${ }^{1} \dagger$

Although genetic control of morphogenesis is well established, elaboration of complex shapes requires changes in the mechanical properties of cells. In plants, the first visible sign of leaf formation is a bulge on the flank of the shoot apical meristem. Bulging results from local relaxation of cell walls, which causes them to yield to internal hydrostatic pressure. By manipulation of tissue tension in combination with quantitative live imaging and finite-element modeling, we found that the slow-growing area at the shoot tip is substantially strain-stiffened compared with surrounding fast-growing tissue. We propose that strain stiffening limits growth, restricts organ bulging, and contributes to the meristem's functional zonation. Thus, mechanical signals are not just passive readouts of gene action but feed back on morphogenesis.

$\mathrm{T}$ he plant shoot apical meristem is composed of two regions, the slow-growing central region, which contains the stem cell niche, and the surrounding periphery, where cells divide rapidly and new organs are initiated (1-4). New organ primordia initiate at accumulation points of the plant hormone auxin (5-7). In addition to triggering gene regulatory pathways, auxin induces cell wall acidification (8), which increases expansin activity (9) that modifies cross-links in the cell wall matrix. Disruption of auxin signaling suppresses organ initiation, which can be restored by the local application of auxin $(7,10,11)$. Bulging in the meristem flank can also be triggered by local cell wall loosening with expansin $(12,13)$ or pectin methyl-esterase (PME) $(14,15)$. These bulges can develop into normal organs, which suggests that a mechanical signal is involved in primordium differentiation. Additional support for mechanical signals in this pathway comes from the recent hypothesis that stress in the cell wall is the signal that orients the

${ }^{1}$ Institute of Plant Sciences, University of Bern, Altenbergrain 21, CH-3013 Bern, Switzerland. ${ }^{2}$ CNRS - Laboratoire de Biogenèse Membranaire, UMR5200, 146 rue Leo Saignat, F-33076 Bordeaux, France. ${ }^{3}$ Department of Biology, University of Fribourg, Chemin de Musée 10, CH-1700 Fribourg, Switzerland.

*These authors contributed equally to this work.

tTo whom correspondence should be addressed. E-mail: richard.smith@ips.unibe.ch microtubule network and the PIN-FORMED 1 (PIN1) auxin transporter $(16,17)$. Yet despite the accumulating evidence for an instructive role for mechanical signals in organogenesis, the mechanical properties of the shoot apex have only recently begun to be explored $(15,18)$. Here, we examine both the elastic and plastic properties of the shoot apex and link them to growth dynamics.

Tomato vegetative shoot apices were imaged at 11-hour intervals by confocal microscopy in order to monitor their growth. Images were analyzed with MorphoGraphX (19) (Fig. 1) to compute relative changes in cell surface area (Fig. 2). primordium; $M$, meristem, Scale bars, $40 \mu \mathrm{m}$.
Cell surface expansion was $25 \%$ on average in the central region and between 45 and $80 \%$ on average in the periphery, depending on the stage of development of the adjacent primordium. The boundary region between the primordium and the meristem displayed little growth. Our data closely resembled growth patterns in other species (1-4).

In order to examine meristem material properties, we induced tissue deformation by manipulating turgor pressure with osmotic treatments using mannitol and $\mathrm{NaCl}$. Experiments started by adapting the samples in solutions of $0.2 \mathrm{M}$ osmotically active molecules. Subsequent immersion in hypo-osmotic medium $(0 \mathrm{M})$ resulted in a relative increase in total surface area of $6 \pm 2 \%$ $(n=20)$. The treatment revealed regional differences, with cells in the central and boundary regions expanding less than those in the periphery (Fig. 3B, fig. S1A, and fig. S2B). Deflation in hyperosmotic solution $(0.4 \mathrm{M})$ resulted in average shrinkage of $6 \pm 2 \%(n=17)$. The relative area decrease was high for cells at the apex summit and variable on the flank (Fig. 3C, fig. S1B, and fig. S2C). The effects were independent of the type of osmolyte used. In order to distinguish between elastic and plastic deformations resulting from hypo-osmotic treatments, we performed sequential treatment with $0 \mathrm{M}$ medium followed by a return to $0.2 \mathrm{M}$. Whereas the hypoosmotic treatment resulted in a $7 \pm 1 \%(n=5)$ total area increase, after returning to $0.2 \mathrm{M}$ solution, the apices were irreversibly expanded by $2 \pm$ $1 \%$. Therefore, the total expansion after an increase in turgor pressure is primarily an elastic response.
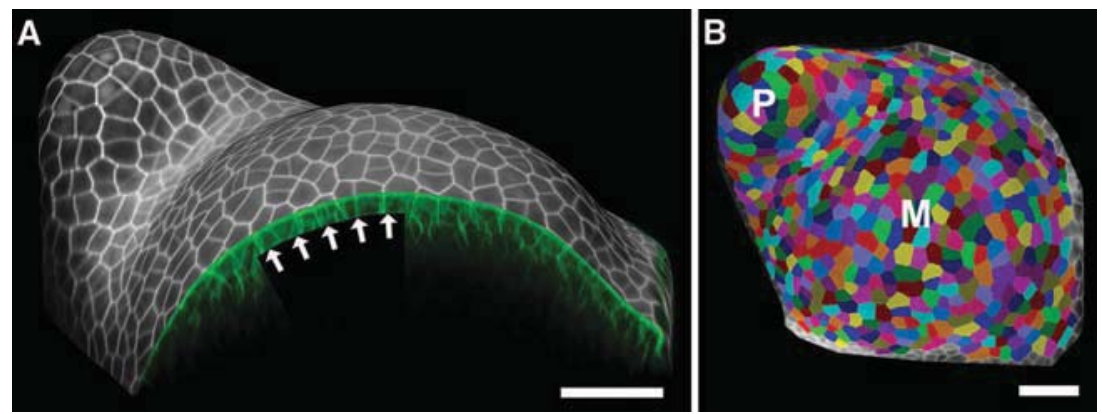

Fig. 1. Quantitative analysis of tissue deformation with MorphoGraphX (www.MorphoGraphX.org). (A) Cell wall signal from the epidermal layer was projected onto a curved surface mesh of the apex. (B) The surface is then segmented into cells and used to track local tissue deformation. P, youngest 
Sequential hypo- to hyperosmotic treatments were then used to track inflation and deflation patterns on the surface of the same apices (Fig. 3, B and C, and fig. S2, B and C). At the apex summit, the same cells that expanded by only $4 \pm 3 \%$ when treated with pure water shrank by $9 \pm 3 \%$ upon plasmolysis (268 cells, three apices). In the periphery, relative areal cell expansion was significantly higher $(9 \pm 5 \%)$ than the shrinkage ( $4 \pm 4 \%)$ (535 cells, three apices). Comparison between deflation and inflation for individual cells shows that cells from the central region and immediate surroundings consistently shrink more than they expand (Fig. 3, D and E; fig. S2, D and E; and fig. S3).

To test whether the observed mechanical properties of the cell wall correlate with functional zones of the shoot apex, we compared growth

Fig. 2. Patterns of growth in tomato shoot apex. Three apices at consecutive stages of primordium development. (A) Gaussian curvature (red, positive; blue, negative). (B) Propidium iodide (PI) signal (red) combined with pDR5::VENUS expression (green) in the epidermis. (C) Heat maps of relative surface area increase over 11 hours of growth (color bar: percentage increase). Arrows indicate the site of next primordium initiation. Scale bars, $40 \mu \mathrm{m}$.
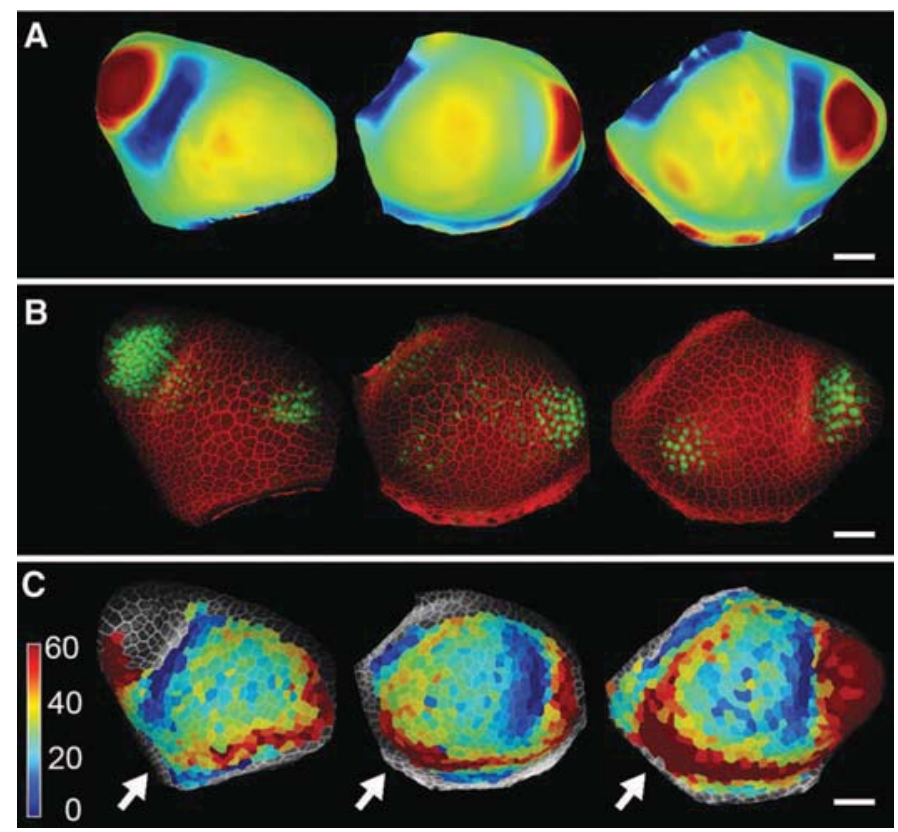

with osmotically induced expansion and shrinkage in apices at similar stages of development (Fig. 3 and fig. S2). The regions of slow and fast growth coincided with areas defined by elastic properties (Fig. 3, A to D and fig. S2, A to D). The results show that surface growth rates, which vary in the functional zones of the meristem, correspond to very different elastic behavior.

The pressure inside cells increases linearly with the osmotic potential; thus, a uniform increase or decrease in extracellular osmotic potential will raise or lower the pressure in all cells equally, barring osmoregulation. Changing the osmolarity by $0.2 \mathrm{M}$ is equivalent to modifying the pressure by $\sim 5$ bars $(0.5 \mathrm{MPa})$. If the elastic behavior was linear, we would expect the cells to expand and shrink roughly by the same amount. In the slow-growing areas, cells shrank more than they expanded (by a factor of about 2), which showed a strongly nonlinear elastic behavior (see supplementary material 4.3). This type of mechanical behavior, known as strain stiffening, is ubiquitous in biomaterials (20-22).

We explored several hypotheses to explain why fast-growing areas in the peripheral region expand more. At the organ level, mechanical stress is commonly thought to be borne mainly by the tunica, the outermost layer or layers of cells (23-27). This abstraction is supported by transmission electron microscopy (TEM) sections that show the outer cell wall is about seven times as thick as the inner walls (fig. S6). Under this assumption, a uniform material property would predict more stress in the flank because of lower
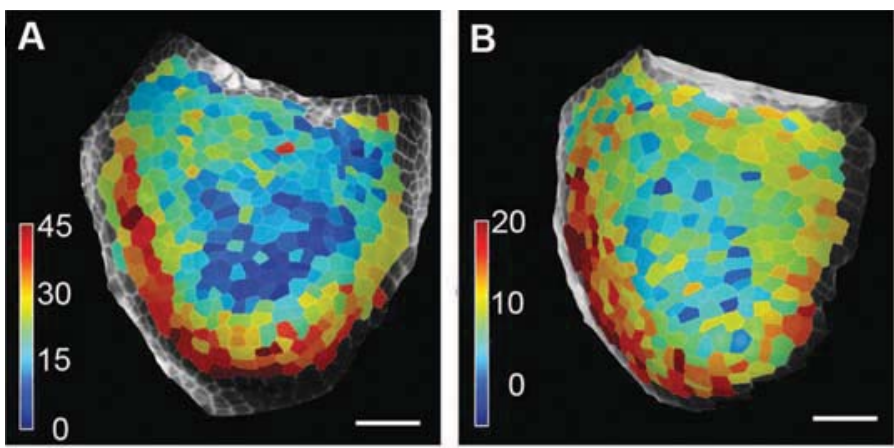

Fig. 3. Comparison between domains of growth and inflation-deflation upon osmotic treatment. (A) Heat maps of relative surface area increase over 11 hours of growth. (B to D) Sequential hypo- to hyperosmotic treatment with $\mathrm{NaCl}$. (B) Heat-map of surface area expansion after hypo-osmotic treatment. (C) Heat-map of surface area decrease after hyperosmotic treatment. Note that some cells in the fastest-growing area were irrecoverably expanded, which resulted in negative shrinkage. (D) Heat map of strainstiffening calculated as percent inflation minus deflation. Cells with lower values (blue) are more strain-stiffened. White dots indicate selected cells corresponding to the slow-growing region at the apex tip. The apex is at a stage similar to that shown in (A). (E) Scattergraph representing the relative surface areal expansion versus shrinkage as a percentage for each cell of the osmotically treated apex. Cells colored in red are indicated by white dots in (D). Dashed line is the boundary where cells shrink as much as they expand. (F and $\mathbf{H})$ Gaussian curvature and (G and I) PI signal (red) combined with pDR5::VENUS expression (green) in the epidermis of the apex used for the growth assay ( $\mathrm{F}$ and $\mathrm{G}$ ) or for osmotic treatment ( $\mathrm{H}$ and $\mathrm{I})$. Gaussian cur-
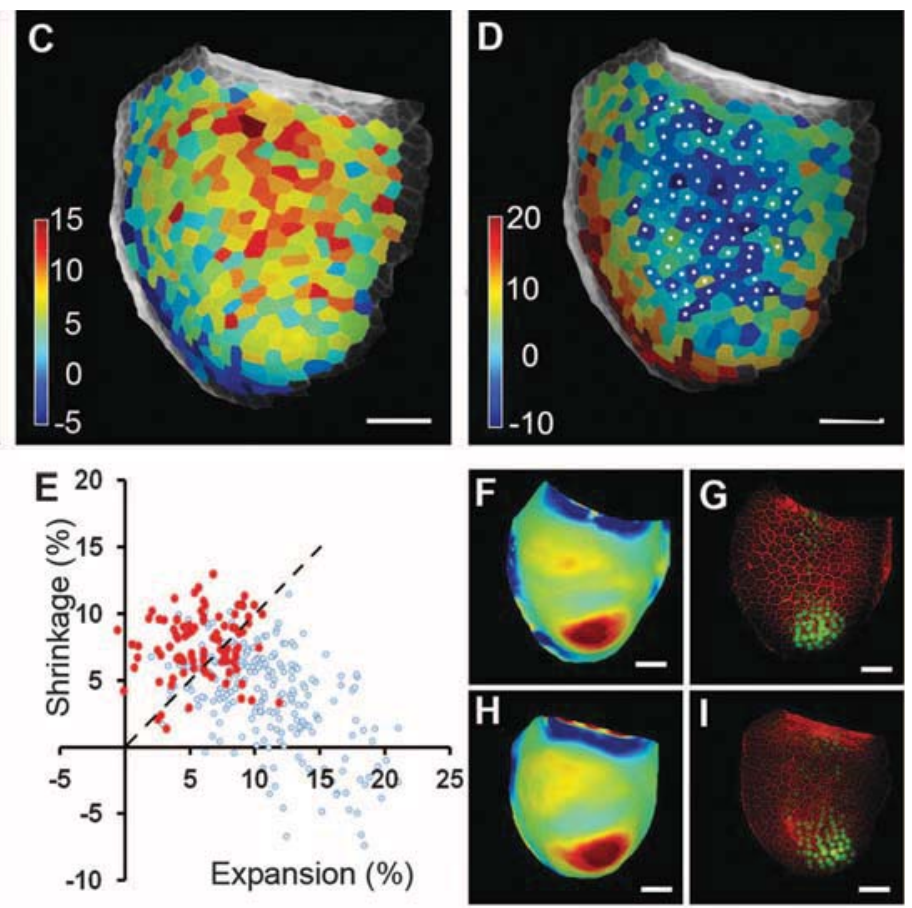

vature (red, positive; blue, negative) was used to compare stages of development between apices with the pDR5::VENUS signal marking the youngest primordia. Color bar (A to D): Relative surface area increase or decrease as a percentage. Scale bars, $40 \mu \mathrm{m}$. 
curvature in this area (fig. S8). This could cause it to expand more relative to the center with increasing pressure. However, decreasing the pressure to zero should also make the peripheral region shrink more, which we did not observe. Thus, geometry alone cannot explain higher expansion in fast-growing areas of the flank.

Next, we asked if differential turgor pressure between regions could explain our results. The deflation assay shows that if there is a turgor difference, then the slow-growing tip region must have higher pressure because it deflates more (Fig. 3C, fig. S1B, and fig. S2C). However, in the sequential hypo- to hyperosmotic treatment, the periphery shrinks more (fig. S4), which indicates that pressure is lower in the central part. Therefore, turgor differences between the regions also do not explain our results.

Although complex hypotheses involving multiple mechanisms might explain the differential behaviors of the fast- and slow-growing regions, we suggest that the data can be explained by simply assuming different strain-stiffening behaviors in the two regions. The following analogy shows how our model works. Suppose two common hair elastic bands of the same length are tied together. If they are stretched from the ends, the load acting on both bands is equal (Fig. 4A). One band, which we call heavier, is harder to expand than the other for small deformations. At first, the lighter band will stretch more; however, at some point, the lighter elastic is strained beyond its linear range of deformation, and its stiffness increases greatly. If we continue to stretch the bands further, the heavier band stretches more easily than the thinner one. During the shift, there exists a crossover point where the stiffness of the two bands is actually equal.

We propose a similar scenario for the shoot apex. The slow-growing apex summit behaves as the light band does, whereas the fast-growing area of the flank behaves like the heavier one. Under normal turgor pressure, the apex is close to the crossover point, where the central region actually becomes harder to expand than the periphery. This intuition can be verified by using a mechanical simulation of the shoot apex using the finite-element method (FEM) (19). We represent the apex as a hemispherical shell of constant thickness and impose two regions of different elastic behavior (Fig. 4B). The material in the center is softer than that in the periphery for small deformation; however, it becomes stiffer for higher strains. The material in the periphery remains in its linear range of deformation for the strain observed (Fig. 4, D and E, and fig. S7). The osmotic experiments were then simulated by increasing and reducing the pressure inside the shell (Fig. 4C). The model was able to explain experimental results by using a simple assumption - a strain-stiffening material that is "heavier" in the periphery (Fig. 4, D and E). As in the experiments, the center shrank more than the flank when we reduced the pressure, whereas it expanded less than the flank when we increased the pressure (Fig. 4, F and G).

Our results show that there is a link between the elastic behavior of the shoot apex and its growth and organogenesis. We find that slow-growing areas of the apex tip are substantially strain-stiffened compared with fast-growing areas in the flank. Although the peripheral region is dynamic, with growth patterns and material properties that change on the basis of the developmental stage of primordia, the central region is consistently slow growing and strain-stiffened. Differential growth between regions may be a direct result of their elastic properties that is, cells must be able to deform elastically in order to grow. Our data support this hypothesis; they suggest that expansion in slow-growing areas, including the central region, is limited by substantial strain-stiffening.

Recent work using atomic force microscopy microindentation techniques showed that cell walls in plasmolysed meristems were stiffer in the central part than at the flanks (18) or primordia (15). Although these studies largely measure compressibility of cell walls, modifying cell turgor pressure allows us to directly assess cell wall stretch under different levels of tension. We expect the response to tension and compression to be different in a reinforced soft material such as the cell wall, where in-plane tension is highly relevant for growth.

We have measured surface deformation, and the simplest interpretation of the results is based on the assumption that the surface layers of cells control growth in the shoot apex (23-27). There are, however, other possible interpretations. For example, mechanical variations in inner tissues may influence the surface deformability. Recently, the subepidermal tissue in the areas of incipient primordia has been reported to be easier to compress than the rest of the meristem (15). Although we did not detect a distinct behavior on these regions on the surface in our experiments, differential surface deformation seen in osmotic assays likely reflects the combined mechanical behavior of the several outermost layers of cells.

Although traditional models relate growth to stress (28), it appears that differential growth in the shoot apex is not because of differential stress, but rather changes in other parameters, such as plasticity or the threshold of stress required for yielding. This has implications for organogenesis and could reinforce the insensitivity of the central region to auxin $(7,11,29)$ or to exogenous application of the wall-modifying enzyme PME (14). Differences in elastic properties may in turn be reinforced by the cell wall
A

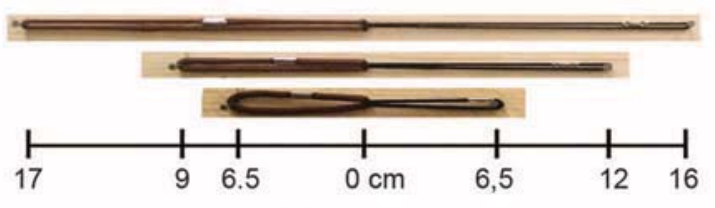

B

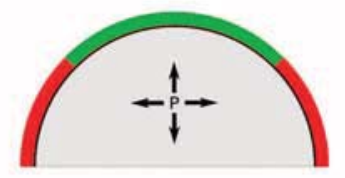

Fig. 4. Mechanical model of the shoot apex. (A) A pair of hair elastic bands, one heavier (left) and one lighter (right), used to demonstrate differential nonlinear elastic behaviors. (B) The shoot apex abstracted as a hemispherical shell. Central material (green) is lighter than the flank material (red). (C) Pressure against areal expansion for the two materials. Both materials have the same stiffness (slope) at the crossover point, close to 5 bar (0.5 MPa). (D and E) FEM simulation of the apex compared with osmotic treatments ( $F$ and $\mathbf{G}$ ). Upon inflation (D), the central area expands less than the flank as in hypo-osmotic treatment (F). Upon deflation (E), the central area shrinks more as in hyperosmotic treatment (G). Color bars: Area increase or decrease as a percentage. Scale bars, $40 \mu \mathrm{m}$.
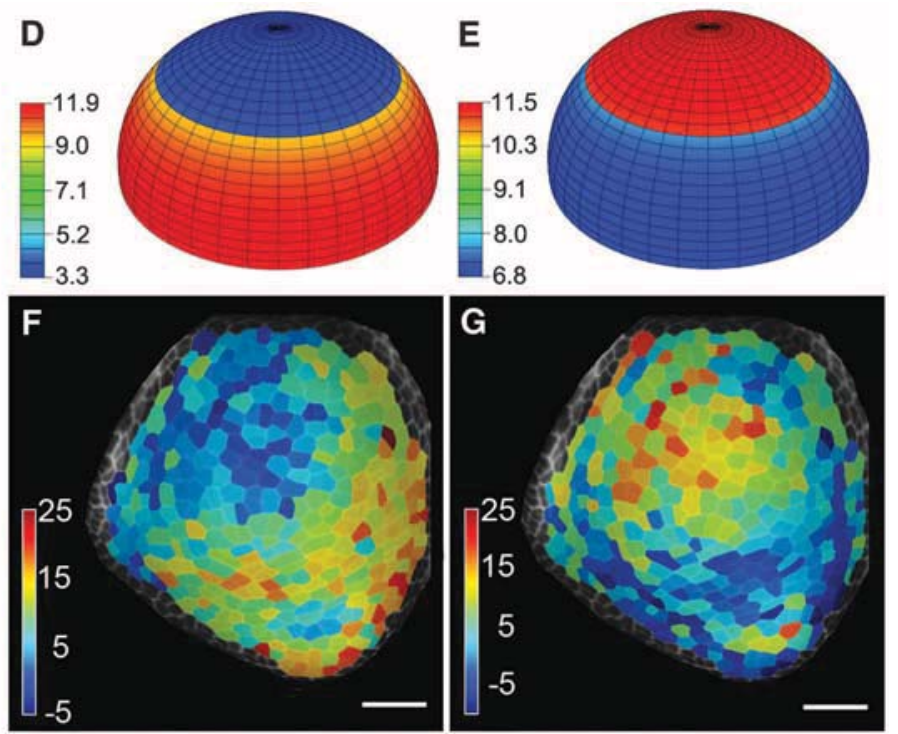
loosening needed for growth. Increased wall remodeling is expected to change elastic properties, which explains why fast-growing areas in the peripheral region can stay in their linear range of elasticity for larger deformations (20-22); while at the same time, they are stiffer than slowgrowing regions for small strains. Our data suggest that the functional distinction between slow- and fast-growing regions in the shoot apex is not only genetically defined $(30,31)$ but is enhanced by mechanical feedbacks. Such a mechanism would stabilize and protect the critically important stem cell niche from the numerous sources of noise inherent in the chemistry of biological systems.

\section{References and Notes}

1. T. Steeves, I. Sussex, Eds., Patterns in Plant Development (Cambrige Univ. Press, New York, 1988).

2. D. Kwiatkowska, ]. Dumais, J. Exp. Bot. 54, 1585 (2003).

3. O. Grandjean et al., Plant Cell 16, 74 (2004).

4. G. V. Reddy, M. G. Heisler, D. W. Ehrhardt, E. M. Meyerowitz, Development 131, 4225 (2004).

5. S. Vanneste, ]. Friml, Cell 136, 1005 (2009).

6. M. G. Heisler et al., Curr. Biol. 15, 1899 (2005).
7. D. Reinhardt et al., Nature 426, 255 (2003).

8. D. L. Rayle, R. E. Cleland, Plant Physiol. 99, 1271 (1992).

9. D. J. Cosgrove, Nat. Rev. Mol. Cell Biol. 6, 850 (2005).

10. T. Vernoux, ]. Kronenberger, O. Grandjean, P. Laufs, J. Traas, Development 127, 5157 (2000).

11. D. Reinhardt, T. Mandel, C. Kuhlemeier, Plant Cell 12, 507 (2000).

12. A. Fleming, S. McQueen-Mason, T. Mandel, C. Kuhlemeier, Science 276, 1415 (1997).

13. S. Pien, ]. Wyrzykowska, S. McQueen-Mason, C. Smart, A. Fleming, Proc. Natl. Acad. Sci. U.S.A. 98, 11812 (2001).

14. A. Peaucelle et al., Curr. Biol. 18, 1943 (2008).

15. A. Peaucelle et al., Curr. Biol. 21, 1720 (2011).

16. M. G. Heisler et al., PLoS Biol. 8, e1000516 (2010).

17. O. Hamant et al., Science 322, 1650 (2008).

18. P. Milani et al., Plant J. 67, 1116 (2011).

19. Materials and methods are available as supporting material on Science Online

20. C. Storm, J. J. Pastore, F. C. MacKintosh, T. C. Lubensky, P. A. Janmey, Nature 435, 191 (2005).

21. S. E. Whitney, M. G. Gothard, ]. T. Mitchell, M. J. Gidley, Plant Physiol. 121, 657 (1999).

22. R. R. Vincent, D. N. Pinder, Y. Hemar, M. A. Williams, Phys. Rev. E Stat. Nonlin. Soft Matter Phys. 76, 031909 (2007).

23. ]. Dumais, C. R. Steele, J. Plant Growth Regul. 19, 7 (2000).

24. J. M. Selker, G. L. Steucek, P. B. Green, Dev. Biol. 153, 29 (1992).
25. R. Vandiver, A. Goriely, Philos. Transact. A Math. Phys. Eng. Sci. 367, 3607 (2009).

26. V. Mirabet, P. Das, A. Boudaoud, O. Hamant, Annu. Rev, Plant Biol. 62, 365 (2011).

27. U. Kutschera, K. J. Niklas, J. Plant Physiol. 164, 1395 (2007).

28. J. A. Lockhart, J. Theor. Biol. 8, 264 (1965).

29. T. Vernoux et al., Mol. Syst. Biol. 7, 508 (2011).

30. R. Sablowski, Curr. Opin. Plant Biol. 10, 639 (2007).

31. R. K. Yadav, T. Girke, S. Pasala, M. Xie, G. V. Reddy, Proc. Natl. Acad. Sci. U.S.A. 106, 4941 (2009).

Acknowledgments: We thank ]. Dumais, A. Fleming, S. Robinson, D. Kwiatkowska, and P. Wojtaszek for reading the manuscript. This work was supported by the Swiss National Science Foundation, SystemsX.ch, and the European Union New and Emerging Science and Technology grant MechPlant. N.N. received European Molecular Biology Organization Long-Term and Roche Research Foundation fellowships.

\section{Supporting Online Material}

Materials and Methods

Figs. $\mathrm{S} 1$ to $\mathrm{S} 8$

References (32-41) 